\title{
Chronic Foot Pain and Foot Solutions in Adults from Different Professions: the I-Corps-National Science Foundation Foot Health Survey.
}

Ngozi D. Mbue ( $\sim$ nmbue@twu.edu )

Texas Woman's University - Houston Center https://orcid.org/0000-0003-1707-0817

Wanyi Wang

Texas Woman's University - Houston Center

Martin G. Rosario

Texas Woman's University

\section{Research}

Keywords: Chronic foot pain, Professions, I-Corps, National Science Foundation

Posted Date: March 31st, 2021

DOI: https://doi.org/10.21203/rs.3.rs-356021/v1

License: (c) (i) This work is licensed under a Creative Commons Attribution 4.0 International License.

Read Full License 


\section{Background}

Approximately $61 \%$ of adults in the United States 21 years and older (135 million people)

experienced foot pain, soreness and discomfort [1]. The most affected are the weight-bearing areas, such as the forefoot (ball) and the rear foot (heel) [1]. These areas are protected by the plantar surface (bottom of the foot) by fat pads, which deteriorates and loosens the protective ability as we age. By the age of 50, people lose as much as their fat padding in the ball and heel areas [1]. In addition to the loss of the fat pad, other physiological changes happen in the feet, such as weakening of muscles, loss of resilience of tendons and ligaments, and spreading of the feet due to weight gain, thereby leading to more soreness and pain that can affect the ability to walk, exercise or engage in recreational activities.

Foot problems is a common concern in most individuals, especially those who stand or walk for prolonged periods of time due to their jobs $[2,3]$. Health care providers, especially nurses and other professions such as athletes, construction and restaurant workers are always on the go, logging miles each day $[4,8]$. Studies show that foot pain contributes to sick leave and early retirement in individuals who stand and walk for prolonged periods of time at home or at their job sites $[5,6,8]$. It is a major cause of nursing staff shortages, which impose a huge pressure on the entire healthcare system. Over the years, many nurses and individuals in similar professions suffer from dilapidating health outcomes due to CFP. Long hours of standing and walking damage and inflame the plantar fascia ligament, which extends from the heel to the toes [8]. According to a recent Japanese study, approximately $36 \%$ of nurses experience lower extremity pain due to prolonged periods on their feet standing and walking during the day [11]. Rates are even higher in nurses who are obese and have been working for over 40 years. Foot pain often contributes to knee and back pain, all of which interfere with the nurse's ability to remain at the beside performing patient care [7,9]. According to a recent study conducted in Ethiopia-Africa, chronic and disabling foot pain affects at least $55.3 \%$ of the general population $[8,10]$. Approximately half $(52 \%)$ surveyed in the United Stated reported sore feet after being on their job all day with $44 \%$ reporting a negative impact on their productivity [12].

Often, athletes are at the highest risk of developing foot and heel pain and it is the most frequent injury in ballet dancers [12-14]. Running/jogging, hiking, playing basketball, fitness walking and dancing are among activities that cause foot issues in those surveyed in the United States [12]. In the military, those who are greater than 40 years old have 3.4 times higher incidence of foot pain related to plantar fasciitis than those in the 20 years-old group [15]. Foot pain related to plantar fasciitis alone accounts for over one million patient visits per year in the United States [16-18]. Construction, as well as restaurant workers, to mention but a few, experience CFP due to high demands such constant walking and lifting objects at their job sites.

Unfortunately, many people deal with foot pain on a daily basis because of their profession without proper solutions or treatments to their foot problems. Foot pain has been associated with increased in age, female sex, higher body max index $[19,20]$, foot pathologies and poor foot habits such as wearing high-heel shoes [21, 22, 23], as well and depression [24]. Prolonged standing and walking are detrimental to the feet, as well as continuous heavy lifting of objects and have been associated with impaired foot function, disability and significant impact on the quality of life [22, 25,26].

Treatment and current solutions to foot and heel pain are largely nonoperative and are managed as outpatients [27]. Treatments include non-steroidal anti-inflammatory drugs, custom-made orthotics, splints and surgery in severe cases, with an estimated cost of treating foot pain between $\$ 500$ and $\$ 20,000$ per person. However, most of these treatments are controversial. For example, the use of nonsteroidal anti-inflammatory drugs (NSIADs) to treat CFP is a widespread practice that has been questioned as most of the foot pain is not an inflammatory process [28], however, the importance of NSIADs in combination with other treatment modalities that may provide short-term pain relief cannot be over emphasized [29]. The pain-relieving effects of localized injections, such as corticosteroids, are short-term; their use must also be weighed against the potential side effects of fat pad atrophy and plantar fascia rupture [27]. Surgeries, which are the final treatment options [27], often do not take care of CFP. Most of these 
treatment options and solutions have various degrees of efficacy; however, they are inadequate for moderate to acute pain in those that stand, lift objects and walk for prolonged periods of time.

Although previous studies have been conducted on foot pain and its impact on the quality of life, the foot health concerns and solutions of those who stand up for prolonged periods at work or at home have not been systematically investigated. Hence, there is a significant unmet need for new treatment options for nurses and those in similar professions that suffer from chronic foot and heel pain due to prolonged standing, walking and lifting objects at their job sites. Embarking on the I-Corps-National Science Foundation (NSF) programs was ideal to illicit information about the current state of foot pain, solutions and potential avenues to explore ways to develop inventions that will address chronic foot pain in individuals from different professions who stand for prolonged periods of time on their feet. The NSF program supports teams of faculty and graduate students as they explore the commercialization of earlystage ideas. I-Corps instructors teach teams to identify product opportunities through their research [30].

The aim of this study was to explore the current state of chronic foot pain, investigate current solutions and treatment options for chronic foot pain, and explore opportunities for future inventions to address chronic foot pain for those who stand for prolonged periods of time on their feet.

\section{Methods \\ Design}

Personal interviews involving open-ended questions to illicit meaningful information from stakeholders about the current state of chronic foot pain, solutions and potential innovations to address CFP were conducted. We conducted predominantly face-to-face interviews and by Zoom since COVID-19 on individuals from various professions, predominantly nurses, restaurant workers, athletes and construction workers, whose jobs required prolonged standing, lifting and walking. To date, our team has attended two regional and one national I-corps NSF program to explore the state of chronic foot pain and solutions in adults from different professions with the intention of developing innovations to address chronic foot pain. Data were obtained from individuals $(\mathrm{N}=119)$, who took part in our interviews.

The NSF launched the Innovation Corps program in 2011 to foster entrepreneurship and facilitate commercialization of NSF-funded technologies [30]. The program supports teams of faculty and graduate students as they explore the commercialization of early-stage ideas. I-Corps instructors teach teams to identify product opportunities through their research. In addition, it assists innovators in creating devices specific to their participants' needs. Each of the I-corps programs comprised seven weeks of intense face-to-face interviews with stakeholders, among other activities.

\section{Participants}

Potential participants were contacted prior to interviews by Zoom, LinkedIn, WhatsApp, Facebook, email and phone calls. To accommodate the participants' schedules, interviews were conducted on the participants' chosen times and days for the interview. Participants were randomly selected from different cities and states in the United States. We visited hospitals, universities, athletic centers, restaurants, nursing organization websites and job construction sites to locate potential individuals. All interview questions were open-ended and lasted for approximately 20-30 minutes.

Inclusion criteria were adults aged 18 years old or older, able to give oral consent to participate in the interviews, and having lived experiences of foot or leg pain. Participants who had not been diagnosed with foot or leg pain were considered. Those with a job that required prolonged standing, walking and lifting were all included in the interviews. Exclusion criteria included not consenting to participate in the interview, not having a job that required prolonged standing, walking or standing and not willing to be interviewed for at least 20 minutes. Ethical approval was obtained from the Woman's Institutional Review Board. Data were collected, de-identified and stored in a secure password-protected personal computer. 


\section{Data Collection:}

Data were gathered using open-ended questions during the individual interviews. Prior to stakeholders' interviews, each team of NSF participants was required to come up with sets of hypotheses to be tested with potential stakeholders. From the list of hypotheses, survey questions were derived and quantified. In addition, demographic information was collected that asked about the pain level, solutions and current treatments of current foot pain. Teams' hypotheses included: i) prolonged standing and walking $>8$ hours a day cause pain in the feet and legs of nurses and other professions; ii) pain relief and prevention contribute to increased productivity and improved quality of life; and iii) current foot pain solutions are not sufficient for those with pain greater than 5 on a scale of zero to ten. Minor topics for discussion were identified using a predetermined interview schedule of questions written prior to individual interviews. Follow-up and the majority of subsequent questions were directed based on the participants' responses to the initial questions. The study questions included: i) How many hours do you spend standing, walking or lifting objects at your job? ii) On a scale of zero to ten, how would you rate your pain while performing your duties at your job? ii) While on your feet or legs do you experience the worst pain when at work or home? iv) What are your problems with regard to prolonged standing or walking at your job or home? and v) Do you use any solution to assist with your pain? All data were de-identified immediately after collection and entered into the Excel software.

\section{Data Analysis:}

Descriptive statistics were first used to report categorical variables using frequencies and percentages. The pain scores were dichotomized into two levels: low pain (0-4) and high pain level (5-10). Crosstabulations using chi-square or Fisher's exact tests were conducted to examine the relationships between the two categories of pain, pain regions, and solutions. Logistic regression was used to examine the relationship between age and various solutions. All data were analyzed using SPSS v25 and statistical $p<.05$ was set at significance.

\section{Results \\ Participants Characteristics}

The data presented in this study comprised of 119 individual interviews. Table 1 presents the demographic information of the participants. The participants were predominantly women $(79 \%)$. The percentages between females and males in participants with a pain level of 5 and above were consistent with the total sample ( $80 \%$ females versus $20 \%$ males). Nearly half of the sample was non-Hispanic white (42\%), followed by non-Hispanic black (13\%), Asian (8\%), and Hispanics (8\%). The majority of the participants were between and 20-50 years of age (83\%), mostly working between 36-40 hours/week (96\%). Participants were mostly nurses (56\%), followed by athletes (12\%) and administrative assistants $(10 \%)$.

Table 1 Frequencies and Percentages on Participants Characteristics

\begin{tabular}{lcc} 
Demographic Characteristics & $\boldsymbol{n}$ & \% \\
\hline Gender & & \\
$\quad$ Female & 94 & 79.0 \\
$\quad$ Male & 25 & 21.0 \\
Gender with pain levels 5-10 & & \\
$\quad$ Female & 24 & 80.0 \\
$\quad$ Male & 6 & 20.0 \\
Race/Ethnicity & &
\end{tabular}




\begin{tabular}{|c|c|c|}
\hline Non-Hispanic Blacks & 15 & 12.6 \\
\hline Asian & 10 & 8.4 \\
\hline Non-Hispanic Whites & 50 & 42.0 \\
\hline Hispanics & 9 & 7.6 \\
\hline Other & 35 & 29.4 \\
\hline \multicolumn{3}{|l|}{ Age } \\
\hline $20-30$ & 32 & 26.9 \\
\hline $30-40$ & 35 & 29.4 \\
\hline $40-50$ & 32 & 26.9 \\
\hline $50-60$ & 14 & 11.8 \\
\hline $60-70$ & 6 & 5.0 \\
\hline \multicolumn{3}{|l|}{ Working Hours } \\
\hline 24 & 2 & 1.7 \\
\hline 30 & 2 & 1.7 \\
\hline 36 & 38 & 31.9 \\
\hline 40 & 76 & 63.9 \\
\hline 50 & 1 & .8 \\
\hline \multicolumn{3}{|l|}{ Profession } \\
\hline Nurses & 67 & 56.3 \\
\hline Athletes & 14 & 11.8 \\
\hline Administrative & 12 & 10.1 \\
\hline Restaurant & 9 & 7.6 \\
\hline Housekeeping & 4 & 3.4 \\
\hline Dietary & 2 & 1.7 \\
\hline Others & 11 & 9.1 \\
\hline
\end{tabular}

\section{Experience with Pain}

Pain was dichotomized into 0-4 and 5-10 (5 representing moderate pain level) on a scale of 0-10. Thirty participants reported a pain level of $\geq 5$. When the pain was grouped into high and low levels of pain (cut off at 5), the results indicated that a greater proportion of Asians $(60 \%)$ had a higher level of pain than non-Hispanic white (14.0\%), Fisher's Exact $=9.17, p=.018$ (see Table 2).

Table 2 Relationships between Pain Score/Level and Race

\begin{tabular}{|c|c|c|c|c|c|c|c|c|c|}
\hline \multirow[b]{2}{*}{ Race } & \multicolumn{3}{|c|}{ Level 0-4 } & \multicolumn{3}{|c|}{ Level 5-10 } & \multirow{2}{*}{$\begin{array}{c}\text { Fisher's } \\
\text { Exact }\end{array}$} & \multirow[b]{2}{*}{$p$} & \multirow[b]{2}{*}{ Cramer's V } \\
\hline & n & $\%$ & & $\mathbf{n}$ & $\%$ & & & & \\
\hline & & & & & & & 9.17 & .018 & .349 \\
\hline Non-Hispanic & & & & & & & & & \\
\hline Blacks & 11 & $73.3 \%$ & $\mathrm{ab}$ & 4 & $26.7 \%$ & $\mathrm{ab}$ & & & \\
\hline Asian & 4 & $40.0 \%$ & $\mathrm{~b}$ & 6 & $60.0 \%$ & $\mathrm{~b}$ & & & \\
\hline Non-Hispanic & & & & & & & & & \\
\hline Whites & 43 & $86.0 \%$ & a & 7 & $14.0 \%$ & a & & & \\
\hline Hispanics & 7 & $77.8 \%$ & $\mathrm{ab}$ & 2 & $22.2 \%$ & $\mathrm{ab}$ & & & \\
\hline
\end{tabular}


Note. For each column category, the proportions with different superscripts differed significantly, $p<$ .05 .

\section{Pain Solutions Comparison and Their Relationships with Age}

Of the 30 participants who reported pain levels above or greater than 5, the most common solution was to change shoes. About $63.3 \%$ of participants changed shoes, $53.3 \%$ used shoe inserts and painkillers, $40.0 \%$ used compression stockings, and only $4(13.3 \%)$ of them did nothing to control their pain.

Nineteen people said the solution was ineffective and none reported that the solution was effective. When comparing the pain solutions with the pain levels, as shown in Table 3, a greater proportion of participants with $\geq 5$ pain levels used shoe inserts, nursing clogs, and painkillers (53.3\%) than those with pain less than $5(27.3 \%), p=.009$. None of the participants with pain levels $5-10$ reported that the solution was effective, whereas $59.4 \%$ of participants with pain levels $0-4$ reported that the solutions were effective, $p<.001$. There were no significant differences in other pain solutions between the two pain levels of the participants.

Then, a series of logistic regressions were used to predict the solution outcomes from age. The results were combined and are presented in Table 4. Younger participants were more likely to report effective solutions than older participants $(O R=.537, p=.007)$. Older participants who thought pain solution was not as good as anticipated experienced the highest pain. Furthermore, older participants were more likely to look for solutions for their pain and use compressions socks regularly than younger participants, but the relationship only reached marginal significance $(p=.051)$, but the difference was not statistically significant.

Table 3 Relationships between Pain Levels and Pain Solutions

\begin{tabular}{|c|c|c|c|c|c|c|c|c|c|}
\hline \multirow[b]{2}{*}{ Variable } & \multicolumn{3}{|c|}{ Level 0-4 } & \multicolumn{3}{|c|}{ Level 5-10 } & \multirow[b]{2}{*}{$\chi^{2}$} & \multicolumn{2}{|r|}{ Cramer's } \\
\hline & $\mathrm{n}$ & $\%$ & & $\mathbf{n}$ & $\%$ & & & $\mathbf{p}$ & V \\
\hline $\begin{array}{l}\text { Compression } \\
\text { stockings }\end{array}$ & & & & & & & .49 & .484 & .064 \\
\hline No & 59 & $67.0 \%$ & $\mathrm{a}$ & 18 & $60.0 \%$ & $\mathrm{a}$ & & & \\
\hline Yes & 29 & $33.0 \%$ & $\mathrm{a}$ & 12 & $40.0 \%$ & $\mathrm{a}$ & & & \\
\hline Shoe changes & & & & & & & .07 & .798 & .024 \\
\hline No & 30 & $34.1 \%$ & $\mathrm{a}$ & 11 & $36.7 \%$ & $\mathrm{a}$ & & & \\
\hline Yes & 58 & $65.9 \%$ & $\mathrm{a}$ & 19 & $63.3 \%$ & $\mathrm{a}$ & & & \\
\hline
\end{tabular}

Shoe insert, nursing clogs, painkillers

No

Yes $64 \quad 72.7 \% \quad$ a

14

6.78

.009

.240

No solutions

$24 \quad 27.3 \% \quad$ a $\quad 16 \quad 53.3 \%$ b

No

$69 \quad 78.4 \% \quad$ a

26

.97

.324

.091 
$\begin{array}{lllllll}\text { Yes } & 19 & 21.6 \% & \text { a } & 4 & 13.3 \% & \text { a }\end{array}$

Solution

effectiveness

$\begin{array}{llrrrrr}\text { No } & 26 & 40.6 \% & \mathrm{a} & 19 & 100.0 \% & \mathrm{~b} \\ \text { Yes } & 38 & 59.4 \% & \mathrm{a} & 0 & 0.0 \% & \mathrm{~b}\end{array}$

Wear

compression

socks regularly

$\begin{array}{lrrrrrr}\text { No } & 38 & 65.5 \% & \text { a } & 10 & 58.8 \% & \text { a } \\ \text { Yes } & 20 & 34.5 \% & \text { a } & 7 & 41.2 \% & \text { a }\end{array}$

$20.81<.001 \quad .501$

$\begin{array}{lll}.26 & .613 \quad .058\end{array}$

Note. For each row category, the proportions with different superscripts differed significantly, $p<.05$

Table 4 Logistic Regressions Predicting Solution and Effectiveness from Age

\begin{tabular}{|c|c|c|c|c|c|c|c|}
\hline \multirow[b]{2}{*}{ Outcomes } & \multirow[b]{2}{*}{$\boldsymbol{\beta}$} & \multirow[b]{2}{*}{$S E$} & \multirow[b]{2}{*}{ Wald } & \multirow[b]{2}{*}{ OR } & \multirow[b]{2}{*}{$p$} & \multicolumn{2}{|c|}{ 95\% CI for OR } \\
\hline & & & & & & $L L$ & $\boldsymbol{U} \boldsymbol{L}$ \\
\hline Compression stockings & -.075 & .17 & .20 & .928 & .658 & 1.294 & 1.399 \\
\hline Shoe changes & -.123 & .17 & .54 & .885 & .464 & .637 & 1.228 \\
\hline $\begin{array}{l}\text { Shoe insert, nursing } \\
\text { clogs, painkillers }\end{array}$ & .163 & .17 & .94 & 1.177 & .333 & .846 & 1.638 \\
\hline No solutions & -.204 & .21 & .92 & .816 & .337 & .538 & 1.236 \\
\hline Solution effectiveness & -.622 & .23 & 7.29 & .537 & .007 & .342 & .843 \\
\hline $\begin{array}{l}\text { Wear compression } \\
\text { socks regularly }\end{array}$ & .441 & .23 & 3.80 & 1.555 & .051 & .998 & 2.422 \\
\hline
\end{tabular}

\section{Pain Regions with Pain Solution}

We examined the regions in relationship to pain solutions and regrouped data into "foot and heel," "heel and leg," and "none." Among the participants who reported pain, there was a significant relationship between regions and shoe changes, Fisher's exact $=4.16, p=.048$. As seen in Table 5, all participants who had heel and leg pain used shoe change solution, while only $61.6 \%$ of participants who reported foot and heel pain used shoe change solution. However, no significant relationships were found between the pain regions and other pain solutions. 
Table 5 Relationships between Pain Regions and Pain Solutions

\begin{tabular}{|c|c|c|c|c|c|c|c|c|c|}
\hline \multirow[b]{2}{*}{ Variable } & \multicolumn{3}{|c|}{ Foot and heel } & \multicolumn{3}{|c|}{ Heel and leg } & \multirow{2}{*}{$\begin{array}{c}\text { Fisher's } \\
\text { Exact }\end{array}$} & \multirow[b]{2}{*}{$p$} & \multirow{2}{*}{$\begin{array}{r}\text { Cram } \\
\text { r's I }\end{array}$} \\
\hline & $n$ & $\%$ & & $n$ & $\%$ & & & & \\
\hline Compression stockings & & & & & & & 1.62 & .416 & .132 \\
\hline No & 53 & $61.4 \%$ & a & 6 & $85.7 \%$ & a & & & \\
\hline Yes & 33 & $38.4 \%$ & $\mathrm{a}$ & 1 & $14.3 \%$ & a & & & \\
\hline Shoe changes & & & & & & & 4.16 & .048 & .212 \\
\hline No & 33 & $38.4 \%$ & a & 0 & $0.0 \%$ & $\mathrm{~b}$ & & & \\
\hline Yes & 53 & $61.6 \%$ & a & 7 & $100.0 \%$ & $\mathrm{~b}$ & & & \\
\hline $\begin{array}{l}\text { Shoe insert, nursing } \\
\text { clogs, painkillers }\end{array}$ & & & & & & & $<.01$ & 1.000 & .001 \\
\hline No & 49 & $57.0 \%$ & a & 4 & $57.1 \%$ & a & & & \\
\hline Yes & 37 & $43.0 \%$ & $\mathrm{a}$ & 3 & $42.9 \%$ & a & & & \\
\hline No solutions & & & & & & & 1.94 & .338 & .145 \\
\hline No & 67 & $77.9 \%$ & a & 7 & $100.0 \%$ & a & & & \\
\hline Yes & 19 & $22.1 \%$ & a & 0 & $0.0 \%$ & a & & & \\
\hline Solution effectiveness & & & & & & & 1.42 & .392 & .142 \\
\hline No & 38 & $58.5 \%$ & a & 5 & $83.3 \%$ & a & & & \\
\hline Yes & 27 & $41.5 \%$ & a & 1 & $16.7 \%$ & a & & & \\
\hline $\begin{array}{l}\text { Wear compression } \\
\text { socks regularly }\end{array}$ & & & & & & & 2.91 & .153 & .215 \\
\hline No & 36 & $62.1 \%$ & a & 5 & $100.0 \%$ & a & & & \\
\hline Yes & 22 & $37.9 \%$ & $\mathrm{a}$ & 0 & $0.0 \%$ & a & & & \\
\hline
\end{tabular}

Note. For each row category, the proportions with different superscripts differed significantly, $p<.05$

\section{Cost of Products}

Those with moderate-to-high pain scales (greater than 5 on a scale of $0-10$ ) spend approximately $\$ 150$ per pair of shoes using approximately 5-7 pairs of shoes interchangeably, and spending approximately \$500$\$ 700$ per year on new shoes. Participants also indicated spending approximately $\$ 40-50$ per pair of orthotic inserts every three months and spending $\$ 160-\$ 200$, (\$20-\$30 per pair) of compression socks every six months amounting to $\$ 40-\$ 90$ per year. Participants would also experience an increase in overthe-counter pain reliever purchases, such as NSAIDs, or even use opioids for pain relief periodically. 


\section{Discussion}

This is an important study and first to examine the state of chronic foot pain among professions, current solutions and treatment with the intention of designing inventions to address chronic foot pain for those who stand for prolonged periods of time on their feet.

While this study included both men (21\%) and women (79\%), one important finding was a consistently higher prevalence of foot pain in women (80\%) than in men $(20 \%)$. Our study has added to the knowledge of not only examining prevalence, but also pain perception and daily experiences of chronic foot pain in both men and women in various occupations. These differences have been widely reported in previous studies [14, 22, 23,31], and have been attributed to other factors such as foot wearing habits, especially in women and prolonged standing and pain perception $(12,31)$. Women are more likely to report musculoskeletal pain in general [31], and pain perception [31]. In addition to the women reporting more foot pain, other studies have shown that moderate-to-severe foot pain is highly prevalent in a university-educated population and is independently associated with women [7]. Our study included both non-university-educated-individuals in various professions. The impact of prolonged standing, walking and lifting heavy objects on the feet and their relationship to various professions and gender differences need to be adequately investigated.

Due to the nature of many professions, employees have no choice when it comes to meeting the requirements of their jobs. This is true for nurses and other professions such as construction workers and housekeeping to mention, but a few. In the United States, nurses are among the largest sector of the healthcare workforce, with more than 20 million nurses worldwide and over 4 million nurses in the United States [32]. In our study, female nurses aged 30-40 years experienced the highest foot pain compared to nurses in their younger age group. On a standard basis, nurses who work in hospital settings are working 12-hour shifts, and from those interviewed, 80-90\% of those 12 hours are spent on their feet. Addressing foot pain among nurses and other professions such as nursing, is needed considering their current working situations [33].

Another important area of our study was to determine the various chronic foot pain solutions and their effectiveness among our participants. Our main finding was that the participants reported commonly used solutions to their current foot pain. These solutions and treatments consisted of non-steroidal antiinflammatory drugs (ibuprofen), steroids (cortisone), periodic opioids use, plasma injections, splints, special footwear, compression socks and orthotic inserts. However, none of these solutions or treatments have been reported to be effective in addressing long-term chronic foot pain in the long term especially in those experiencing moderate to severe pain. Studies have shown that conservative interventions such as routine foot care, footwear devices and foot orthoses were effective at reducing foot pain and may have assisted in maintaining mobility [34]. The differences in experiences and perceptions of pain relief could be related to the number of hours standing and lifting heavy objects while on one's feet. Most of the participants indicated that their pain level was highest when they were at work and actively on their feet than when they were at home or not involved in prolonged standing or lifting heavy objects. Surprisingly, a few of those interviewed indicated that prolonged sitting also causes foot pain.

The precise definition of foot pain varied among participants in both younger and older participants as well as the reasons for seeking an intervention. Some participants reported their foot pain as "foot discomfort," expected when you stand for prolonged periods of time, while some participants described their pian "as common wear and tear with age." The available evidence from previous studies [34], indicates that foot pain is common and disabling in mostly older people, and we have shown that chronic foot pain is equally prevalent in younger working force. In our study, participants aged 30-40 years old reported higher pain than those in the younger age group. Our data showed that the majority of the participants did not consult a health care practitioner with their chronic foot pain as useful. Most reported that they have learned to live with their pain as normal wear and tear in the body as we age. 
Younger participants preferred not to address their foot pain or to use any support. Older participants addressed their pain and used solutions such as support hoses, inserts and shoes than younger participants. Younger participants reported pain relief compared to older participants. Older participants who thought pain solution not as good as anticipated experienced the highest pain. In addition, older participants wore compression socks more regularly than the younger participants did, because they may have other issues such as varicose veins and a higher frequency of pain-related conditions such as osteoarthritis [31]. We realized that some of the participants did not consult a specialist such as a podiatrist or their primary care providers for their chronic foot pain as urgent. Most indicated that they have learned to live with their pain, as most solutions do not help their chronic foot pain. There have been inconsistent results regarding the reason for consulting a health care practitioner, with some studies indicating that only a minority of older people would consult their health care practitioners with musculoskeletal foot problems, while positive participation in treatment efficacy appears to be the strongest factor in influencing consultation with clinicians [35]. This is an area that requires further investigation.

The prevalence of and solutions to chronic foot pain differed among races. Although the participants comprised $42 \%$ of non-Hispanic Whites, while Asians comprised 8\%, Non-Hispanic Blacks $13 \%$ and Hispanics $8 \%$, it is interesting how each group reported pain level and solutions to their chronic foot pain. For example, $60 \%$ of Asians reported higher pain levels compared to $14 \%$ of non-Hispanic Whites. Asians with low tolerance to pain maybe due to differences in pain perception or pain relief measures. Of the 10 Asians, only one person reported using shoes, inserts, and nursing clogs. Ninety\% of Asians changed their shoes instead of taking pain medications. However, 40\%-55\% of non-Hispanic Blacks reported adequate pain solution. Maybe reasons for the variations in solutions for foot pain may have been related to the nature of the injury and the area of pain. For example, biomechanics, body weight, lack of exercise, prevalence and particular foot pain area near the toes, heel, and middle of the foot may have affected the different reasons for different solutions. Interestingly, there were no significant differences in the types of solutions in different areas by race, which warrants further studies. Relationship of foot pain with other health conditions has been extensively studied with foot pain associated with those who are obese, upper and lower extremities musculoskeletal problems [31]. In our study, the majority reported mostly heel, leg, knee and back pain. Periodically, most nurses reported back and knee pain. Moderate-to-severe foot pain was associated with worse scores for foot function, foot health and health-related quality of life in our study as well as other studies [31, 34].

Educational attainment in the work area does not appear to be protective against moderate-tosevere foot pain. back pain and hip pain, as indicated by the majority of nurses and athletes in our study. Most nurses indicated that they received training and education on ergonomics at the initial stages of their employment by their employers without elaborate efforts to address subsequent chronic foot pain afterwards. This is an area that needs further evaluation to explore options by employers to assist their employees to preventing and addressing chronic foot pain and solutions for their employees.

To our knowledge, this is the first investigation into the experiences of individuals in various professions with daily chronic foot pain who stand, walk and lift heavy objects at their job sites. This is a representative snapshot. By examining the state of chronic foot pain and current solutions, rich information is acquired that will be useful in developing inventions to address chronic foot pain in professions that stand for prolonged periods of time on their feet.

This study has some limitations that warrant further attention.. Participants were from limited professions and regions of the United States; therefore, data may not have been a representative of the general population from other cities, states and countries. Nevertheless, this study provides a solid foundation from which researchers and clinicians can begin to understand the daily experiences of their patients as they access care and find ways to manage foot pain in clinical practice. Future studies should 
focus on collaboration with other clinics or institutions, and recruitment by social media in order to identify a more accurate representation of those with chronic foot pain.

\section{Conclusions}

This study highlights the experiences of those with CFP who stand for prolonged periods of time at their job, solutions and provided potential areas to explore for inventions to address foot pain. There is a consistent result of participants' affirmation of the lack of effective solutions to their chronic foot pain. Future studies should use more consistent measures such as pressure distribution assessment follow by the implementation of tailored shoe insoles in prospective studies to evaluate the impact of prolonged standing, heavy lifting and walking have on foot and potentially effective solutions to address this debilitating CFP. Further investigation such as gait assessment to determine any underlying modifications that might case pain before it becomes a chronic issue is warranted, as many individuals in various occupations suffer from debilitating musculoskeletal pain without adequate pain solutions Qualitative data showed that majority of those experiencing higher (greater than 5) pain levels are abandoning things such as sleep and personal hours, by an average of 1-2 hours after work hours. The majority expressed involvement in some activities after work to care for their pain. These activities included stretching, taping, soaking, massaging and elevating the feet. It was found that the majority of those who are experiencing greater than 5 pain would tolerate and feel more productive at a pain rating of 2 or 3 on a scale of $0-10$. The tolerable pain scale, we also asked, "what would you gain by reducing your pain to your tolerable pain scale?" Participants answers varied from being more productive at work and having a higher quality of family life at home.

List of abbreviations: NSF: National Science Foundation, I-Corps-Innovation Corps; CPF: Chronic foot pain; NSAIDs: Nonsteroidal anti-inflammatory drugs.

\section{Declarations}

Ethics approval and consent for study

All participants provided consent to participate in the survey. Ethical approval was obtained from the Texas Woman's University Investigational Review Board (reference number: IRB FY 2021-136).

\section{Consent for Publication}

Ethical approval was obtained from the Texas Woman's University Investigational Review Board (reference number: IRB FY 2021-136).

\section{Availability of data and materials}

The datasets generated and/or analyzed during the current study are not publicly available due to (Patent Confidentiality), but are available from the corresponding author upon reasonable request.

Competing interests: None

\section{Funding}

This work was supported by grants from the National Science Foundation (Award ID 1954004)-Federal (CFDA \# 47.041 Engineering Grants). 


\section{Authors' contributions}

NM conducted the study and was a major contributor in writing the manuscript, WN analyzed and interpreted the data and contributed to the manuscript writing, MR contributed to the manuscript writing. All authors read and approved the final manuscript.

\section{Acknowledgements}

We would like to thank the participants, Jessica Wade (Entrepreneur Lead), a graduate student in the Family Nurse program at Texas Woman's University, Rosha Joshi, PhD student (Entrepreneur Lead) for interviewing and collecting data. In addition, we thank Dr. Bijan Najafi (I -Corps Mentor). Grateful to all the NSF I-Corps faculty and staff for their directions and assistance in all the NSF regional and national programs. 
